\title{
Isochrones for late-type stars
}

\author{
Pierre Demarque \\ Department of Astronomy, Yale University \\ P.O. Box 208101, New Haven , CT 06520-8101, USA \\ email: pierre.demarque@yale.edu
}

\begin{abstract}
A brief summary of the history of stellar evolution theory and the use of isochrones is given. The present state of the subject is summarized. The major uncertainties in isochrone construction are considered: chemical abundances and color calibrations, and the treatment of turbulent convection in stellar interior and atmosphere models. The treatment of convection affects the modeling of stellar interiors principally in two ways: convective core overshoot which increases evolutionary lifetimes, and the depth of convection zones which determines theoretical radii. Turbulence also modifies atmospheric structure and dynamics, and the derivation of stellar abundances. The symbiosis of seismic techniques with increasingly more realistic threedimensional radiation hydrodynamics simulations is transforming the study of late-type stars. The important case of very low mass stars, which are fully convective, is briefly visited.
\end{abstract}

Keywords. Convection, turbulence, stars: abundances, stars: evolution, stars: late-type, stars: low-mass; stars: oscillations

\section{Introduction}

The determination of stellar ages has been a primary motivation of the study of stellar structure beginning in the late 19th century. Early estimate of stellar ages had relied on gravitational contraction and the release of gravitational energy (the Kelvin-Helmholtz timescale), leading to an apparent conflict with the much longer geological timescale estimates. Although the possible role of nuclear fusion in stellar energy generation was recognized early in the 20th century, the development of wave mechanics and nuclear physics in the 1930's provided the solution to the origin of stellar energy, culminating in the work of Bethe (1939).

In this talk, I wish to focus on some of the uncertainties met in comparing the observational data to the models. There has been much effort in understanding source of uncertainties in fitting the data to isochrones that are considered more or less free of errors. I wish to emphasize the role played by present uncertainties in the underlying physics of stellar evolution. The story of stellar dating parallels that of our understanding the physics of stellar interiors and atmospheres, and future progress will depend on advances in modeling and in new observational tools, such as high precision abundance determinations, parallaxes, interferometric angular diameters and astero-seismology. The reliability of the next generations of isochrones will depend on how well we understand the physics of cool stars.

Early applications of stellar models to the Hertzsprung-Russell diagrams (HRD) of star clusters illustrate this point, drawing an example taken from Chapter 7 in Chandrasekhar's monograph (1939). The author describes a theory that explains misleadingly the differences in the HRD of star clusters of different ages to their internal helium abundance, assumed to be uniform within stars. The major breakthrough came from Öpik (1938) who realized that stars in general do not evolve mixed, and that they 
develop helium rich cores which make them evolve into giant stars. The concept of the main sequence turnoff was born, and with the subsequent discovery of the SchönbergChandrasekhar (1942) limit at core exhaustion. Stars like the Sun were then believed to have a convective core (Cowling 1936). Quantitative estimate of stellar ages at the main sequence turnoff became possible. The evolution of globular cluster stars was outlined in physical terms and sketched as a sequence of static models in the HRD by Hoyle \& Schwarschild (1955). The authors derived a preliminary age of 6.2 Gyr for the globular cluster M3.

The first automatic calculations of stellar evolution are due to Haselgrove \& Hoyle (1956a). They derived an approximate age of 6.5 Gyr for M3 on the basis of 16 evolutionary models (1956b). The construction of grids of stellar evolutionary tracks, from which isochrones are constructed, came in the early and mid-1960's in papers by (Demarque \& Larson 1964; Kippenhahn et al. 1967; Iben 1967) and many others since then.

\section{The last twenty years}

As emphasized in the introduction, recent improvements are the result of advances in input physics motivated, for late-type stars, in major part by studies of the solar interior stimulated by solar neutrino research, and by helioseismology. Indeed helioseismology, by probing the run of sound speed in the core of the Sun, played a key role in the "resolution" of the classical solar neutrino problem (Bahcall et al. 1995, 2001, 2005).

\subsection{Input physics}

Giant strides have been made in the input physics for late-type stars in the last twenty years in the following areas, which are crucial in isochrone construction:

Nuclear reactions. Progress was motivated principally by the solar neutrino research.

Interior opacities. Due to the remarkable agreement of several independent projects (Los Alamos, OPAL, the OPACITY Project), using different conceptual and numerical approaches (Iglesias \& Rogers 1996; Rogers \& Iglesias 1994; Seaton \& Badnell 2004; Seaton 2007), confidence in radiative opacities for the stellar interior has greatly increased.

Low temperature opacities. Low temparature opacities are particularly critical in cool stars. Calculations have been performed by Kurucz (1995), Alexander and Ferguson (1994), Ferguson et al. (2005) and OPAL, extending the range and density of the opacity grids.

Equation of state In both opacities and the equation of state (e.g. OPAL, Rogers et al. 1996), but primarily in the equation of state, because it affects the calculation of the speed of sound most directly, helioseismology has played a central role in stimulating progress.

\subsection{The role of element diffusion}

Helioseismology has also demonstrated the role played by element diffusion in the solar interior, both in the layers just below the convection zone, and in the solar core. As a result, the effects of diffusion are normally included in the construction of the so-called standard solar model (SSM) (Bahcall et al. 1995; Guenther \& Demarque 1997).

Helium and heavy element diffusion are known to affect isochrone ages (Chaboyer et al. 1992), particularly in globular cluster stars which exhibit thin convection zone near the main sequence turnoff due to their low metallicities. The efficiency of diffusion can be inhibited by the presence of turbulence or other internal mixing processes (Chaboyer et al. 1995). Diffusion also plays an important role in the interpretation of lithium abundance 
data (Deliyannis et al. 1990). The effects of helium diffusion are included in the $Y^{2}$ isochrones (Yi et al. 2001; Kim et al. 2002; Demarque et al. 2004).

\subsection{Theoretical isochrones and their applications}

There are now many sources of theoretical isochrones. The reader is referred to proceedings of IAU Symposium 252, held earlier this year in Sanya, China, for a more extensive description of isochrone grids available in the literature. Increasingly, grids of isochrones are being developed for special research purposes. A recent example is the version version 3 of the Yonsei-Yale $\left(Y^{2}\right)$ isochrones, described by Yi et al. (2008) and soon to be posted on the web, which includes a larger range of helium abundances and improved equation of state in the low mass stars.

Isochrones are used in the interpretation of color-magnitudes of resolved stellar systems and clusters (Gallart et al. 2008; Lee et al. 2005; Norris 2004; Piotto et al. 2005; Sarajedini et al. 2007; Stetson et al. 2004), unresolved systems (Bruzual \& Charlot 2003; Yi 2003) and field stars (Pont \& Eyer 2005).

\section{Main uncertainties in isochrone construction}

While enormous progress has been achieved in the constitutive microscopic physics of stellar models for late-type stars, serious uncertainties still remain in a number of areas central to the construction of isochrones. I discuss here briefly three main uncertainties. These uncertainties are (1) convective core overshoot, (2) the depth of convection zones and the mixing length theory, and (3) atmospheric structure and dynamics, and the effect on abundance determinations and color transformations, which are known to be particularly deficient for cool stars, where atmospheric convection is known to dominate. All three of these uncertainties are primarily affected by our inadequate knowledge of turbulent convection.

\subsection{Isochrones and convective core overshoot}

Convective core overshoot beyond the boundary of the convective core set by the Schwarzschild criterion (1906) lengthens the core hydrogen burning phase in stellar evolutionary tracks. On isochrones, the shape of the turnoff region is changed. Figure 1 shows the effect. The resulting turnoff luminosity function is very different. Evolutionary timescales both near the main sequence and on the giant branch are also modified. The consequence of core overshoot for the integrated spectral energy distribution of a simple stellar population (SSP) has been discussed by Yi (2003).

\subsection{Isochrones and the mixing length uncertainties}

On an isochrone, the radii of all stellar models with a convective envelope is affected by the choice of the mixing length parameter $\alpha$, a free parameter usually calibrated on solar models. Primarily for simplicity and convenience, theoretical isochrones are constructed using a constant vale of $\alpha$ during evolution. There is really neither theoretical nor empirical justification for assuming that $\alpha$ should remain constant along an evolutionary track (Demarque et al. 1992; Robinson et al. 2004). In fact, there is evidence that the effective $\alpha$ is a function of both position in the HRD and depth within stars. The sensitivity of the giant branch position to the choice of $\alpha$ within the framework of the MLT is illustrated in Figure 2. The effect on the spectral energy distribution of the corresponding SSP has also been discussed by Yi (2003). 


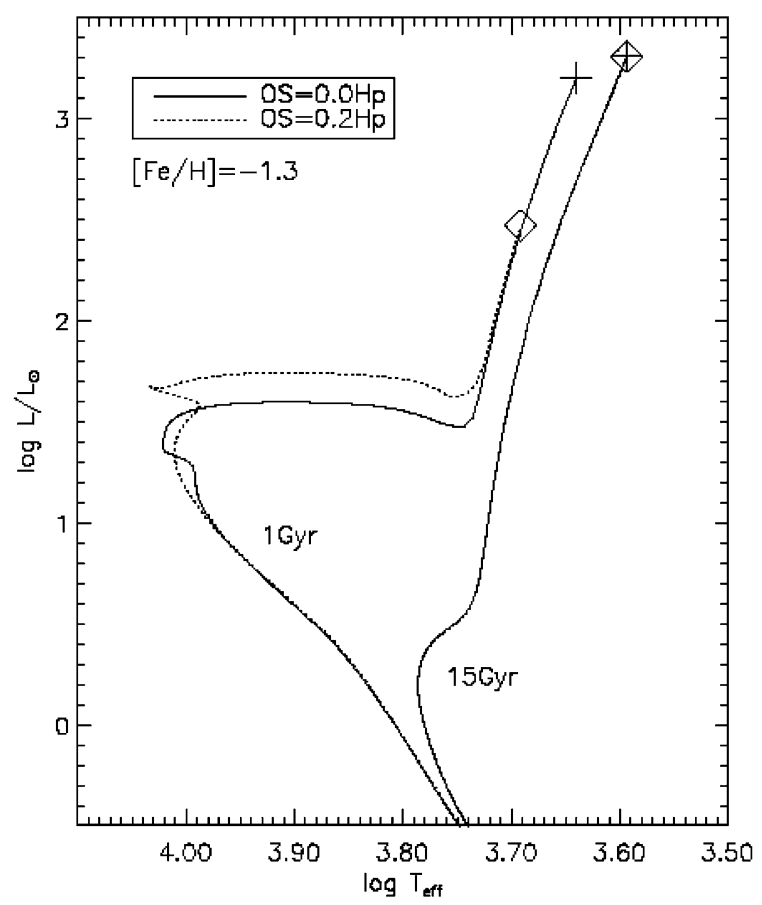

Figure 1. Convective core overshoot (OS) affects the shape of the isochrone and the hydrogen burning lifetime (Yi 2003).

\subsection{Modeling atmospheric structure and dynamics}

The crucial importance of a physically consistent treatment of turbulence in the atmospheres of late-type stars is illustrated by the recent revision of solar abundances, based on the use of a 3D simulation of the solar atmosphere (Asplund et al. 2005). A detailed discussion of the implications of this revision for stellar physics and helioseismology is given by Basu \& Antia (2008).

Colors are also sensitive to atmospheric structure and dynamics; it is well known that present calibrations are very unreliable for cool stars (see e.g. Yi 2003). An extensive discussion of the differences between color calibrations by several authors can be found in VandenBerg \& Clem (2003).

\section{New research tools}

Next generation isochrones will require improvements in the description of turbulent convection, and turbulence in regions which are formally in radiative equilibrium (according to the Schwarzschild criterion), such as overshoot regions.

More physically consistent turbulence in model stellar atmospheres is needed for high precision abundance estimates. A more realistic treatment of core overshoot is needed to derive reliable ages for intermediate mass and massive stars. In particular, reliable spectral dating of distant galaxies depends on this information (Yi et al. 2000). The depth of convection zones and the precise position of the giant branch for different chemical compositions depend on a description of convection to replace the MLT. In the case of fully convective stars at the low mass end, the derivation of the M-L-R relation is determined by physical conditions near the surface (Limber 1958; Allard et al. 1997; Chabrier \& Baraffe 2000). 


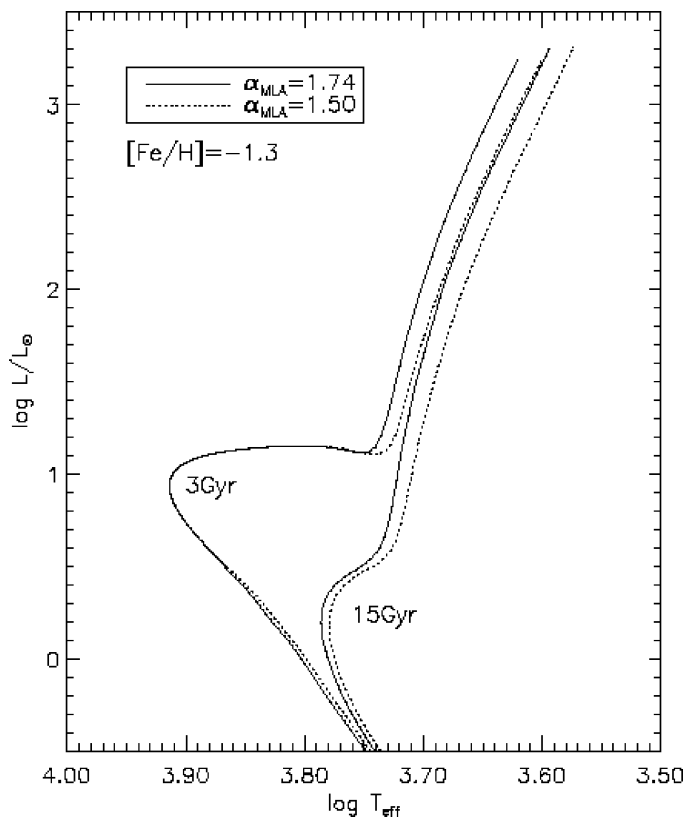

Figure 2. The mixing length approximation parameter $\alpha$ affects the shape of isochrones. A larger value of $\alpha$ makes the position of the giant branch bluer (Yi 2003).

Two new research tools are now available to study the effects of turbulent convection in stars. On the theoretical side, it is now possible to carry out physically realistic threedimensional simulations of radiation hydrodynamics (3D RHD) (Chan \& Sofia 1989; Nordlund \& Dravins 1990; Kim et al. 1995; Stein \& Nordlund 1998; Kim \& Chan 1998; Robinson et al. 2003, 2004). More recently, simulations for metal-poor dwarf stars have also been carried out (Jung et al. 2007; Ludwig et al. 2008). On the observational side, seismology offers radically new ways to probe the deep interior of stars, by means of the WIRE, MOST, CoRoT and Kepler space missions, and of ground-based networks such as SONG. Seismic techniques are particularly well suited to determine precisely the position of radiative-convective interfaces and chemical composition discontinuities (Basu \& Antia 1995).

\subsection{Some of what we can learn from $3 D$ RHD}

There is a wide variety of basic stellar physics problems that can be addressed using three dimensional radiation hydrodynamics simulations. Some of the most relevant are listed below:

- Physically realistic surface boundary conditions for late-type stars (the entropy jump and the convection zone depth).

- Improved model atmospheres for abundance determinations and color calibrations.

- Effects of turbulence on $p$-mode frequency shifts in stellar outer layers

- The excitation of $p$-modes (Samadi et al. 2007).

- viscous dissipation due to turbulence in stellar convection zones(e.g. orbital circularization in binary stars and exoplanet orbital decay) (Zahn \& Bouchet 1989; Penev et al. 2007).

- The properties of convection, which when combined with rotation, drive magnetic activity in late-type stars.

- The formation of shocks leading to mass loss. 


\subsection{Parameterization of turbulent convection}

One of the challenge is the parameterization of 3D simulations to $1 \mathrm{D}$ and its implementation into 1D stellar models (Lydon et al. 1992). From the 3D simulations, one can derive the following pieces of information:

- The form of the $T(\tau)$ relation in the atmosphere.

- The specific entropy in the deep convection zone. This quantity determines the depth of the convection zone, and the position of the tachocline at the interface between the convection zone and the radiative envelope.

- The specific entropy is determined by the structure of the outer convection zone $(\mathrm{CZ})$ and of the highly superadiabatic layer (SAL). In the language of the mixing length theory (MLT), $\alpha$ is the parameter that is used to tweak the specific entropy.

- The sound speed (and the $p$-mode frequencies) are sensitively affected by the outer envelope and atmospheric structure.

- The parameterization should describe as faithfully as possible the turbulent velocity field to which absorption line profiles are sensitive. The simulation is thus capable of providing a measure of the microturbulence, which is a free parameter in conventional 1D stellar atmosphere modeling.

An example of simple parameterization of turbulence is given in the next section.

\subsection{Including turbulence in stellar models}

A method to incorporate the effects of turbulence into the outer layers of one-dimensional (1D) stellar models has been implemented by Li et al. (2002) in the stellar evolution code YREC (Demarque et al. 2008). The approach is based on a formalism due to Lydon \& Sofia (1995). It requires a detailed three-dimensional hydrodynamical simulation (3D RHD) of the atmosphere and highly superadiabatic layer of the star (Robinson et al. 2003). The basic idea is to extract from the velocity field of the $3 \mathrm{D}$ simulation three important quantities: the turbulent pressure, the turbulent kinetic energy and the anisotropy of the flow. Implementation into a 1D stellar model thus requires two additional parameters, i.e. $\chi$, the specific turbulent kinetic energy, and $\gamma$, which reflects the flow anisotropy. These parameters, which modify the hydrostatic equilibrium equation and the internal energy equation, must be introduced in a thermodynamically self-consistent way. As a result, they also change the adiabatic and convective temperature gradients, as well as the energy conservation equation.

Figure 3 illustrates the effect on $p$-mode frequencies of incorporating parameterized turbulence into the standard solar model (SSM). An application to the subgiant $\eta$ Boo is shown in Figure 4 where the resulting echelle diagram is shown (Straka et al. 2006).

\subsection{What we can learn from seismology}

Seismology provides uniquely sensitive probes of stellar interiors, primarily using the properties of acoustic ( $p$-modes) known to be excited in the Sun and late-type stars. In particular, seismology provides:

- Age estimates from a consideration of small and large spacings of the observed p-modes (Christensen-Dalsgaard 1988)(the C-D diagram);

- Detailed information about convective core size and core overshoot (Straka et al. 2005; Mazumdar et al. 2006);

- A measure of the convection zone depth (Christensen-Dalsgaard et al. 1991);

- Sensitive tests of the structure of the outer convective layers (Rosenthal et al. 1999);

- Precise estimates of the envelope helium abundance (Basu \& Antia 1995). 

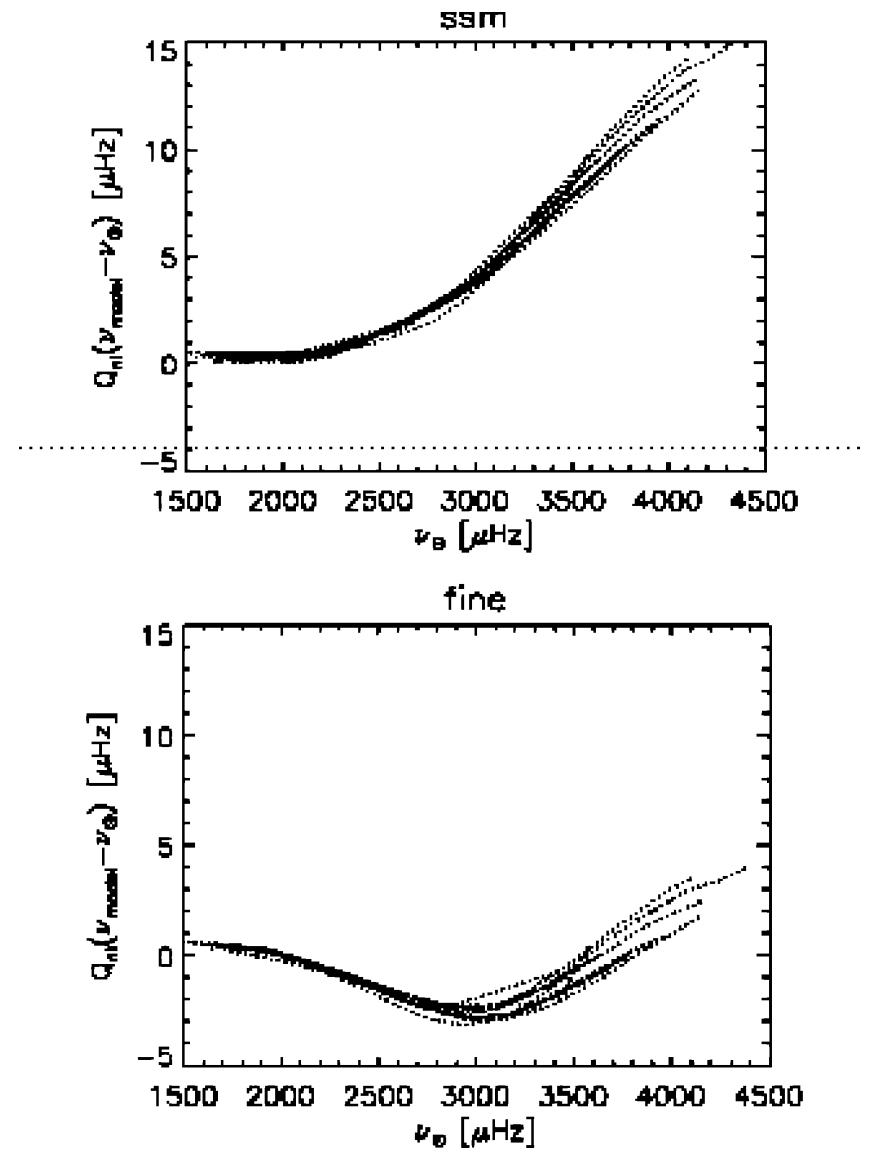

Figure 3. Difference between observed and computed $p$-mode frequencies for a SSM without (top) and with (bottom) turbulence.

One must emphasize here that seismic techniques are complementary to other observational techniques, and cannot replace them. For sun-like stars, seismic techniques are most powerful when used in conjunction with other observations, i.e. when estimates of mass, metallicity, parallax, angular diameter, are also available.

\section{An extreme case: the very low mass stars}

The very low mass stars present a challenging problem. Because they have fully convective interiors, they obey a mass-luminosity-radius (M-L-R) relation, and their central conditions depend sensitively on their surface boundary conditions Limber 1958). As a result, the important independent age indicator, the lithium depletion boundary (LDB) (Burke et al. 2004 is sensitive to details of the atmospheric structure. The 3D RHD simulations by Ludwig (2006) indicate that the chemical equilibrium is complex. Interior models yield masses that do not agree with observations of binary stars by Henry (2004) and Hillenbrand \& White (2004). 


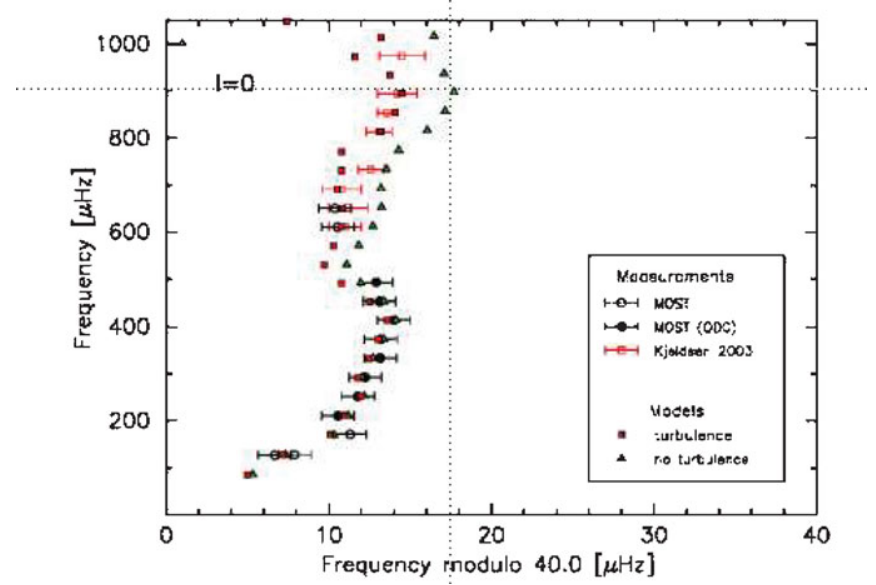

Figure 4. Echelle diagram for a calibrated model of the subgiant $\eta$ Boo with and without the effects of turbulence. The data are taken from Kjeldsen et al. (2003) and the MOST satellite at the lowest frequencies (Guenther 2007).

\section{Future generation isochrones}

This review focuses on some major uncertainties in the construction of stellar evolutionary tracks and isochrones for late-type stars.

More specifically, these uncertainties are (1) the color transformations used to convert the position of stars in the theoretical HRD to their positions in the observational plane; (2) convective core overshoot which principally affects evolutionary lifetimes near the main sequence, but also advanced evolutionary phases; and (3) the treatment of convection in the outer layers of the convection zone. All three of these uncertainties have at their root our insufficient understanding of turbulent convection and its interaction with radiation.

In the extreme case of very low mass stars, which are fully convective, the treatment of convection in the outer layers affect sensitively the M-L-R relation. As a result, masses derived from theoretical isochrones are extremely uncertain. The position of the lithium depletion boundary is also affected.

The theoretical and observational tools are now available to address these problems. On the theoretical side, grids of physically realistic numerical 3D simulations of radiation hydrodynamics can now be constructed. From these, physically realistic model atmospheres will yield more accurate abundances and calibrated spectral energy distributions. At the same time appropriate parameterizations are being devised that will create the stellar evolutionary models for future generation isochrones. On the observational side, the advent of seismic techniques, both space based or in ground-based networks of telescopes distributed around the globe, are beginning to provide the necessary tests of the models. We note that in order to be effective, asteroseismic data will have to be analyzed in conjunction with the high precision astrometric observations and spectroscopic data.

\section{Acknowledgements}

I am especially grateful to S. Yi for his communications in the preparation of this paper. 


\section{References}

Alexander, D. R. \& Ferguson, J. W. 1994, ApJ, 437, 879

Allard, F., Hauschildt, P. H., Alexander, D. R., \& Starrfield, S. 1997, ARAE A, 35, 137

Asplund, M., Grevesse, N., \& Sauval, A. J. 2005, ASPC, 336, 25

Bahcall, J. N., Pinsonneault, M. H., \& Wasserburg, G. J. 1995, RvMP, 67, 781

Bahcall, J. N., Pinsonneault, M., \& Basu, S. 2001, ApJ, 555, 990

Bahcall, J. N., Basu, S., Pinsonneault, M., \& Serenelli, A. M. 2005, ApJ, 618, 1049

Basu, S. \& Antia, H. M. 1995, MNRAS, 276, 1402

Basu, S. \& Antia, H. M. 2008, Phys. Rep., 457, 217

Bethe, H. 1939, Phys. Rev., 55, 434

Burke, C. J., Pinsonneault, M. J., \& Sills, A. 2004, ApJ, 604, 272

Bruzual, A. G. \& Charlot, S. 2003, MNRAS, 344, 1000

Chaboyer, B., Deliyannis, C. P., Demarque, P., Pinsonneault, M. H., \& Sarajedini, A. 1992 ApJ, 388,372

Chaboyer, B., Demarque, P., Guenther, D. B., \& Pinsonneault, M.H. 1995, ApJ, 446, 435

Chabrier, G. \& Baraffe, I. 2000, ARA\&A, 38, 337

Chan, K. L. \& Sofia, S. 1989, ApJ, 336, 1022

Chandrasekar, S. 1939, An Introduction to the Study of Stellar Structure (Chicago: U. of Chicago Press), Chap. 7

Christensen-Dalsgaard, J. 1988, IAUS, 123, 295

Christensen-Dalsgaard, J., Gough, D. O., \& Thompson, M. J. 1991, ApJ, 378, 413

Cowling, T. G. 1936, MNRAS, 96, 42

Deliyannis, C. P., Demarque, P., \& Kawaler, S. D. 1990, ApJS, 73, 21

Demarque, P. R. \& Larson, R. B. 1964, ApJ, 140, 544

Demarque, P., Green, E. M., \& Guenther, D. B. 1992, AJ, 103, 1501

Demarque, P., Woo, J.-H., Kim, Y.-C., \& Yi, S. K. 2004, ApJS 155, 667

Demarque, P., Guenther, D. B., Li, L. H., Mazumdar, A., \& Straka, C. W. 2008, Ap\&SSS, 316, 31

Ferguson, J. W. et al. 2005, ApJ, 623, 585

Gallart, C. et al. 2008, ApJ, 682, 89

Guenther, D. B. \& Demarque, P. 1997, ApJ, 484, 937

Guenther et al. 2007, CoAst, 151, 5

Haselgrove, C. B. \& Hoyle, F. 1956a, MNRAS, 116, 515

Haselgrove, C. B. \& Hoyle, F. 1956b, MNRAS, 116, 527

Henry, T. J. 2004, ASPC, 348, 159

Hillenbrandt, L. A. \& White, R. J. 2004, ApJ, 604, 741

Hoyle, F. \& Schwarzschild, M. 1955, ApJS, 2, 1

Iben, I, Jr. 1967, ARAESA, 5, 57

Iglesias, C. A. \& Rogers, F.G. 1996 ApJ, 464, 943

Jung, Y. K., Kim, Y.-C., Robinson, F. J., Demarque, P., \& Chan, K. L. 2007, ASPC, 362, 306

Kim, Y.-C, Fox, P. A., Demarque,P., \& Sofia, S. 1995, ApJ, 442, 422

Kim, Y.-C. \& Chan, K. L. 1998, ApJ, 496, 121

Kim, Y. -C., Demarque, P., Yi, S. \& Alexander, D. R. 2002, ApJS, 144, 259

Kippenhahn, R., Weigert, A., \& Hofmeister, E. 1967, Meth. Comp. Phys., 7, 129

Kjeldsen, H. et al. 2003, AJ, 126, 1483

Kurucz, R. L. 1995, $A S P C, 81,583$

Lee, Y.-W. et al. 2005, ApJ, 621, L57

Li, L. H., Robinson, F. J., Demarque, P., Sofia, S., \& Guenther, D. B. 2002, ApJ, 567, 1192

Limber, D. N. 1958, ApJ, 127, 387

Ludwig, H.-G., Allard, F., \& Hauschildt, P. H. 2006, A\&A, 459, 599

Ludwig, H.-G., González Hernández, J. I., Behara, N., Caffau, E., \& Steffen, M. 2008, AIPC, 990,268

Lydon, T. J., Fox, P. A., \& Sofia, S. 1992, ApJ, 397, 701

Lydon, T. J. \& Sofia, S. 1995, ApJS, 101, 357 
Mazumdar, A., Basu, S., Collier, B. L., \& Demarque, P. 2006, MNRAS, 372, 949

Nordlund, A. \& Dravins, D. 1990, A\& A, 228, 155

Norris, J. E. 2004, ApJ, 612, 25

Öpik, E. 1938, Pub. Obs. Astr. de l'Univ. de Tartu, vol. 30, no. 3

Penev, K. et al. 2007, ApJ, 655, 1166

Piotto, G., Villanova, S., Bedin, L. G. et al. 2005, ApJ, 621, 777

Pont, F. \& Eyer, L. 2005, ESASP, 576, 187

Robinson, F. J., Li, L. H., Demarque, P., Sofia, S., Kim, Y.-C., \& Guenther, D. B. 2003, MNRAS, 340,923

Robinson, F. J., Demarque, P., Li, L. H., Sofia, S., Kim, Y.-C., Chan, K. L., \& Guenther, D. B. 2004, MNRAS, 347, 1204

Rogers, F. J. \& Iglesias, C. A. 1994, Science, 263, 50

Rogers, F. J., Swenson, F. J., \& Iglesias, C. A. 1996, ApJ, 456, 902

Rosenthal, C. S., Christensen-Dalsgaard, J., Nordlund, Å, Stein, R. F., \& Trampedach, R. 1999, $A \& A, 351,689$

Samadi, R. et al. 2007, A\&A, 463, 294

Sarajedini, A., et al. 2007, AJ, 133, 290

Schönberg, M., \& Chandrasekar, S. 1942, ApJ, 96, 161

1906, Nachr. Kön. Preus. Akad. Wissenschaften 195, 41

Seaton, M. J. \& Badnell, N. R. 2004, MNRAS, 354, 457

Seaton, M. J. 2007, MNRAS, 382, 245

Stein, R. F. \& Nordlund, A. 1998, A\&A A, 499, 914

Stetson, P. B. et al. 2004, PASP, 116, 1012

Straka, C. W., Demarque, P., \& Guenther, D. B. 2005, ApJ, 629, 1075

Straka, C. W., Demarque, P., Guenther, D. B., Li, L. H., \& Robinson, F. J. 2006, ApJ, 636, 1078

VandenBerg, D. A. \& Clem, J. L. 2003, AJ, 126, 778

Yi, S. et al. 2000, ApJ, 533, 670

Yi, S., Demarque, P., Kim, Y. C., Lee, Y-W., Ree, C.-H., Lejeune, T., \& Barnes, S. 2001, (ApJS), 136, 417

Yi, S. K. 2003, ApJ, 582, 202

Yi, S. K., Kim, Y.-C., Demarque, P., Lee, Y.-W., Han, S.-I., \& Kim, D. G. 2008, IAUS, 252, 413

Zahn, J.-P. \& Bouchet, L. 1989, A\&A, 223, 112 


\section{Discussion}

S. YI: Where would the inadequateness of the mixing length convection approximation be more critically affecting? Would isochrone fits be affected much?

P. Demarque: The lowest masses would be most obviously affected because they have deep convective zones. In the more massive stars, core overshooting in the core-H-burning and core He- burning phases would affect evolutionary lifetimes most critically and would also have an impact on the advanced phases of evolution.

E. Jensen: Could you comment on any changes from your new simulations in terms of predictions of Li depletion for low-mass stars?

P. Demarque: I hope that we will have useful results in the next two or three years. We have completed the parallelization of the simulation code, and are improving the treatment of radiative opacities in the atmospheric layers. The improvements will be not only in predicting Li line strengths in the atmospheres, but also in calculating the depth of the convective overshoot in the interiors.

B. WEAVER: Modeling microturbulence has been causing me problems in a stellar atmosphere synthetic spectrum simulation. Would the effect on spectra increase with increasing mass?

P. Demarque: Yes, it seems to be the case. The results we have show that the relative importance of turbulence increases with stellar mass on the main sequence and with decreasing gravity on the giant branch. 

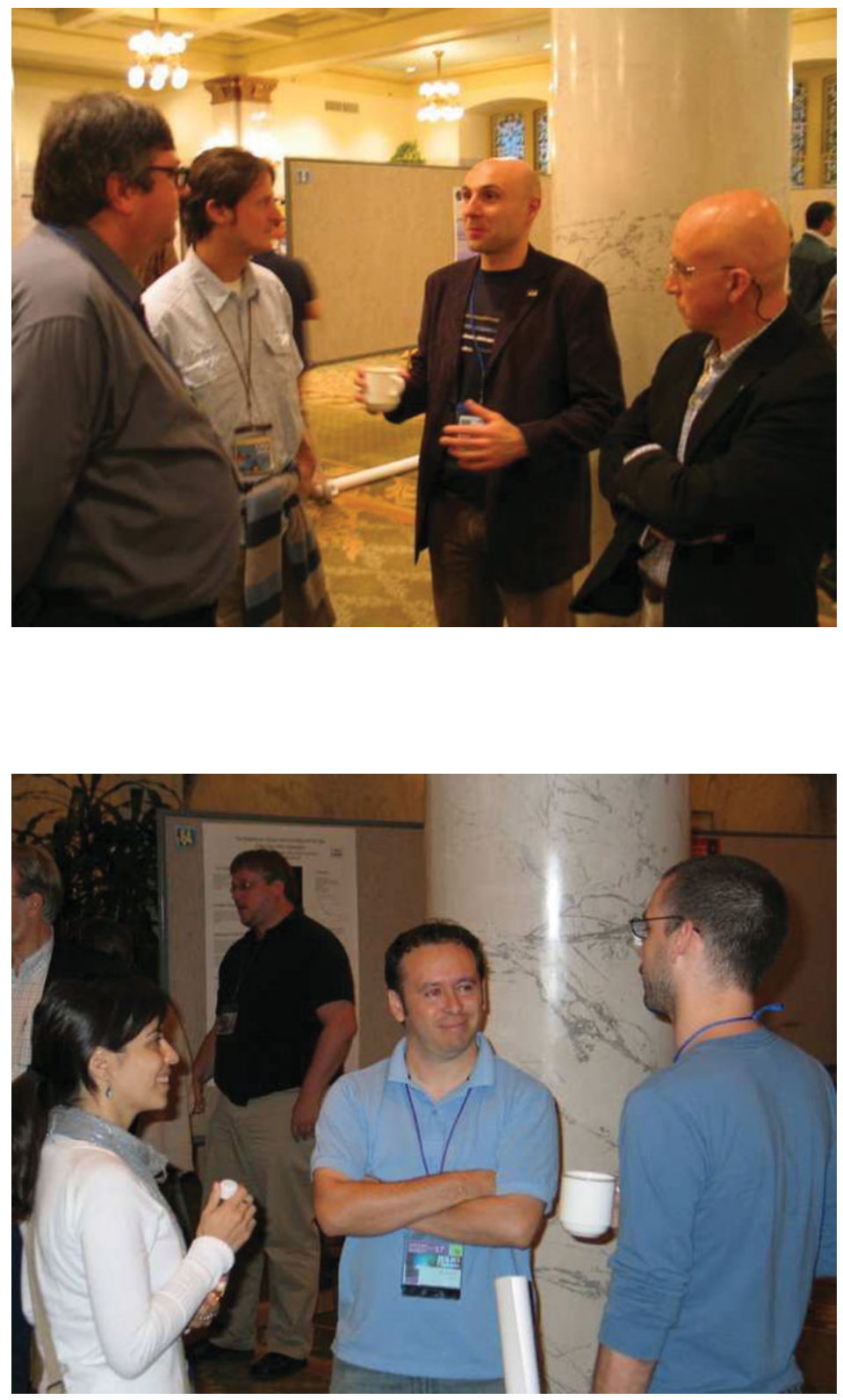